\title{
ON A CHARACTERISTIC FEATURE OF THE POSITIVE LOGICS
}

\author{
KATUZI ONO and JIRO ITO
}

In this short note, we would like to point out that the following property (called ASSUMPTION REMOVABILITY in the present paper) is characteristic of the positive logics, the primitive logic LO, the positive predicate logics LP (intuitionistic) and LQ (classical) $)^{1)}$ :

Assumption Removability. If any proposition (s, can be deduced from some assumption $\mathfrak{A}$ having no primitive notions in common with $(5$, then $\mathbb{C}$ is also provable without any assumption.

This is surely a characteristic property of these positive logics, because the proposition does not hold in the logics LJ (intuitionistic predicate logic), LK (lower classical predicate logic), LM (minimal predicate logic, intuitionistic), and $\mathbf{L N}$ (minimal predicate logic, classical) ${ }^{2}$. One can realize this easily by the pair of example propositions $\rightarrow(A \rightarrow A)$ and $\rightarrow B$. Although $\rightarrow B$ is surely deducible from $\rightarrow(A \rightarrow A)$ in any one of these logics, and moreover, $\rightarrow(A \rightarrow A)$ has no primitive notions in common with $\rightarrow B$, we can never assert that $\rightarrow B$ is provable without any assumption. On the other hand, the ASSUMPTION REMOVABILITY holds for the positive logics as shown later.

LO and LP can be formulated in Gentzen's manner as the sub-logic of Gentzen's LJ having logical constants $\rightarrow$ and ( ) only and the sub-logic of $\mathbf{L J}$ having logical constants $\rightarrow, \wedge, \vee,()$, and ( $\exists$ ), respectively. Also, LQ can be formulated in Gentzen's manner as the sub-logic of Gentzen's LK having logical constants $\rightarrow, \wedge, \vee,()$, and $(\exists)^{3}$. We can easily see that any sequent provable in any one of these positive logics can be proved in the same logic

Received March 30, 1966.

1) As for LO, LP and LQ, see Ono [6]. See also Curry [2] and Lorenzen [5]. Curry refers to $\mathbf{L P}$ and $\mathbf{L Q}$ by $\mathbf{L A}$ and $\mathbf{L C}$ in [2], respectively.

2) As for $\mathbf{L M}$ and $\mathbf{L N}$, see Johansson [4] (Minimalkalkül), Ono [6], and Curry [2]. Curry refers to $\mathbf{L N}$ by $\mathbf{L E}$ in [2].

3) As for Gentzen's LJ and LK, see Gentzen [3]. 
by making use of sequents $\Gamma \mid-\Delta^{1)}$ of non-vacant $\Delta$ only. We can also see that Gentzen's cut-elimination theorem ${ }^{5)}$ holds for any one of these logics.

Now we prove ASSUMPTION REMOVABILITY with respect to the positive logics. Namely, let $\mathbf{L}$ be any one of the positive logics LO, LP, or LQ formulated in Gentzen's manner, and let $₫$ be a proposition deducible in the logic $\mathbf{L}$ from the assumption $\mathfrak{A}$ having no primitive notions in common with $\mathfrak{s}$. Then, the sequent $\mathfrak{A} \mid-(5$ is also provable in $\mathbf{L}$, and by virtue of Gentzen's cut-elimination theorem, $\mathfrak{A} \mid-\mathbb{S}$ can be proved by a proof $I I$ without making use of cuts.

For any sequent $I \mid-\Delta$ in $\Pi$, new sequents $\Gamma_{\mathfrak{A}} \mid-\Delta \mathfrak{A}$ and $\Gamma_{\mathfrak{C}} \mid-\Delta_{\mathfrak{C}}$ are defined by the following:

$\Gamma_{\mathfrak{P}}\left(\right.$ or $\left.\Gamma_{\mathfrak{S}}\right)$ is the sequence of all the propositions in $\Gamma$ which have at least one primitive notion in common with $\mathfrak{A}$ (or with (5). $\Delta_{\mathfrak{A}}$ as well as $\Delta_{\mathfrak{C}}$ is defined similarly.

Now, we call any sequent $\Gamma \mid-\Delta$ in $I$ an $\mathfrak{Q}$-sequent (or a (5-sequent) if and only if $\Gamma_{\mathfrak{A}} \mid-\Delta_{\mathfrak{A}}$ (or $\Gamma_{\mathfrak{C}} \mid-\Delta_{\mathfrak{C}}$ ) is provable in $\mathbf{L}$. Evidently, any fundamental sequent of $\Pi$ is an $\mathfrak{H}$-sequent or a $($-sequent, and any sequent deduced from an $\mathfrak{U}$-sequent or a pair of $\mathfrak{H}$-sequents (a $\mathfrak{5}$-sequent or a pair of $\mathfrak{S}$-sequents) in $\Pi$ is an $\mathfrak{A}$-sequent (a $(5$-sequent).

Moreover, we can show easily that any sequent deduced from a pair of an $\mathfrak{H}$-sequent and a (5-sequent in $\Pi$ is also an $\mathfrak{H}$-sequent or a (5-sequent ${ }^{6)}$. To show this, we have only to check the following three kinds of inferences:

$$
\begin{aligned}
& \frac{\Gamma-\Delta, \mathfrak{F} \quad \Gamma \mid-\Delta, \mathbb{B}}{\Gamma \mid-\Delta, \mathfrak{F} \wedge \mathbb{B}}, \\
& \frac{\Gamma, \mathfrak{F}|\Delta, T, \mathbb{B}|-\Delta}{\Gamma, \mathfrak{F} \vee \mathbb{B} \mid-\Delta}, \\
& \frac{\Gamma|-\Delta, \mathfrak{F} \Gamma, \mathbb{B}|-A}{\Gamma, \mathfrak{F} \rightarrow \mathbb{B} \mid-\Delta, \Lambda}
\end{aligned}
$$

$\widetilde{F} \wedge \mathbb{B}$ in the first inference, as well as $\mathfrak{F} \vee \mathbb{B}$ in the second inference, as well as $\tilde{\mho} \rightarrow \mathbb{B}$ in the third inference, is either a proposition having no primitive notions in common with $\mathfrak{U}$ or a proposition having no primitive notions in

4) We employ the notation $\Gamma \mid-\Delta$ in place of Gentzen's notation $\Gamma \rightarrow \Delta$, because we use $\rightarrow$ as the logical constant IMPLICATION. In Gentzen [3], IMPLICATION is denoted by $=$.

5) The HAUPTSATZ of Gentzen [3].

6) As for the inference schemes for sequents, see Gentzen [3]. 
common with $(5$.

First case : $\mathfrak{F} \wedge \mathbb{B}$ in the first inference (or, $\mathfrak{F} \vee \mathbb{B}$ in the second inference, or $\tilde{F} \rightarrow \mathbb{S}$ in the third inference) have no primitive notion in common with $\mathfrak{A}$. By the supposition, either $\Gamma \mid-\Delta, \widetilde{F}$ or $\Gamma \mid-\Delta, \mathbb{B}$ in the first inference (or, either $\Gamma, \mathfrak{F} \mid-\Delta$ or $\Gamma, \mathbb{B} \mid-\Delta$ in the second inference; or, either $\Gamma \mid-\Delta, \tilde{F}$ or $\Gamma, \mathbb{B} \mid-\Lambda$ in the third inference) is an $\mathfrak{U}$-sequent. Hence, $\Gamma_{\mathfrak{A}} \mid-\Delta_{\mathfrak{A}}$ in the first inference $\left(\Gamma_{\mathfrak{Q}} \mid-\Delta_{\mathfrak{Q}}\right.$ in the second inference, either $\Gamma_{\mathfrak{Q}} \mid-\Delta_{\mathfrak{A}}$ or $\Gamma_{\mathfrak{A}} \mid-\Lambda_{\mathfrak{A}}$ in the third inference ) must be provable in $\mathbf{L}$. Accordingly, $\Gamma \mid-\Delta, \mathfrak{F} \wedge \mathbb{B}$ in the first inference, as well as $\Gamma, \mathfrak{F} \vee|\mathbb{S}|-\Delta$ in the second inference, as well as $\Gamma, \mathfrak{F} \rightarrow|\mathbb{S}|-\Delta, \Lambda$ in the third inference (For LO and LP, $\Delta$ must be vacant, so $\Gamma_{\mathfrak{A}} \mid-\Delta \mathfrak{A}$ can not be provable. Hence, $\Gamma_{\mathfrak{A}} \mid-\Lambda_{\mathfrak{A}}$ must be provable by assumption. For $\mathbf{L} \mathbf{Q}, \Gamma_{\mathfrak{A}} \mid-\Delta_{\mathfrak{A}}, \Lambda_{\mathfrak{A}}$ can be deduced from any one of $\Gamma_{\mathfrak{A}} \mid-\Delta_{\mathfrak{A}}$ and $\Gamma_{\mathfrak{X}} \mid-\Lambda_{\mathfrak{A}}$, and moreover, at least one of these sequents must be provable by assumption.) is an $\mathfrak{A}$-sequent.

Second case : $\mathfrak{F} \wedge \mathbb{B}$ in the first inference (or, $\mathfrak{F} \vee \mathbb{B}$ in the second inference ; or, $\mathfrak{F} \rightarrow(\mathbb{S}$ in the third inference) have no primitive notions in common with $(5$. Also in this case, we can prove quite similary as in the first case that $\Gamma \mid-\Delta, \hat{\vartheta} \wedge \mathbb{B}$ in the first inference, as well as $\Gamma, \mathfrak{F} \vee(\mathbb{S} \mid-\Delta$ in the second inference, as well as $\Gamma, \tilde{V} \rightarrow|\mathbb{S}|-\Delta, \Lambda$ in the third inference is a $(\mathcal{S}$-sequent.

Accordingly, we can conclude that $\mathfrak{U} \mid-\mathfrak{C}$ is also an $\mathfrak{U}$-sequent or a $\mathfrak{S}$-sequent. However, $\mathfrak{U} \mid-$ can never be proved in $\mathbf{L}$ as having been remarked, so $\mid-(\widehat{S}$ must be provable in $\mathbf{L}$.

Remark. According to the interpolation theorem of Craig (for the lower classical predicate logic) and Schütte (for the intuitionistic predicate logic) ${ }^{i)}$, either $\neg \mathfrak{U}$ or $(5$ must be provable in any one of these logics as far as $\mathfrak{A} \rightarrow \mathfrak{b}$ is provable in it for any pair of propositions $\mathfrak{A}$ and $\mathfrak{5}$ containing no primitive notions in common. According to our assertion for positive logics, we can say further that $\leqslant$ must be provable in the corresponding case of any one of positive logics.

\section{REFERENCES}

[1] Craig, W.: Linear reasoning. A new form of the Herbrand-Gentzen theorem, J. Symb. Log., 22 (1957), 250-268.

[2] Curry, H. B.: Foundations of mathematical logic, New York (1963).

7) See Craig [1] and Schütte [7]. 
[3] Gentzen, G.: Untersuchungen über das logische Schliessen, Math. Z., 39 (1934), 176210, 405-431.

[4] Johansson, I.: Der Minimalkalkül, ein reduzierter intuitionistischer Formalismus, Compositio Math., 4 (1936), 119-136.

[5] Lorenzen, P.: Einführung in die operative Logik und Mathematik, Berlin-GöttingenHeidelberg, (1955).

[6] Ono, K.: On universal character of the primitive logic, Nagoya Math. J., 27-I (1966), 331-353.

[7] Schütte, K.: Der Interpolationssatz der intuitionistischen Prädikatenlogik, Math. Ann., 148 (1962), 192-200.

Mathematical Institute,

Nagoya University 